\title{
Multi-modality Therapy of Hepatic Metastases from Colorectal Carcinoma: Optimal Combination of Systemic Chemotherapy with Radio-embolization
}

\author{
Esme J. Hill1,2 and Ricky A. Sharma ${ }^{1,2 *}$
}

${ }^{1}$ Department of Oncology, Cancer Research UK-Medical Research Council Gray Institute for Radiation Oncology \& Biology, Old Road Campus Research Building, University of Oxford, Oxford OX3 7DQ, UK

${ }^{2}$ Department of Oncology, Churchill Hospital, Oxford Radcliffe Hospitals NHS Trust, Oxford OX3 7LJ, UK

\begin{abstract}
The commonest cause of death from advanced colorectal cancer is disease progression of hepatic metastases. A number of technologies are in clinical development to improve local control of liver metastases and potentially improve overall survival. Radio-embolization $(\mathrm{RE})$ is a technique for administering resin or glass microspheres that contain yttrium-90 to unresectable primary or secondary hepatic malignancies internally via the liver's arterial supply in a single procedure. Clinical trials of RE used with concomitant radiosensitizing chemotherapy have shown promising results in patients with metastatic colorectal cancer. In this article, the evidence base for combining RE with systemic chemotherapy in the first line therapy of metastatic colorectal cancer is appraised and the scientific rationale for combining RE with chemotherapy in first and subsequent lines of therapy is outlined. Clinical trials of RE and chemotherapy currently recruiting patients with metastatic colorectal cancer are discussed in detail and practical recommendations offered on how best to combine RE and systemic chemotherapy.
\end{abstract}

Keywords: Chemo-radiation therapy; Selective internal radiotherapy; Oxaliplatin; Irinotecan

Abbreviations: RE: Radio-Embolization

\section{The Clinical Problem of Hepatic Metastases in Colorectal Carcinoma}

Despite major advances in the systemic treatment of metastatic colorectal carcinoma, the 5 year survival rate remains disappointingly low at approximately $7 \%$ [1]. Median survival for this patient group is currently in the range 1.5 to 2.5 years, and depends on continuation of systemic therapy for much of the patient's remaining life. Of all patients with metastatic colorectal cancer, $20-30 \%$ have liver-only metastases and approximately $50 \%$ of recurrences following resection of the primary tumour are confined to the liver alone. Surgical resection of hepatic metastases is the treatment of choice, where possible, but this is feasible for less than $15 \%$ of patients at presentation [2]. For the subset of patients with metastatic colorectal cancer in whom surgical resection can be achieved, the 5 year overall survival probability is 30 $40 \%$, with $20 \%$ of patients achieving long-term cure [3]. Patients with unresectable liver predominant metastases have increasingly become a focus of interest for improving survival for patients with metastatic colorectal cancer. This prioritization is due to the outcomes from Phase II studies utilising downstaging neo-adjuvant chemotherapy in which $10-20 \%$ of patients with inoperable liver disease have been converted to curative resection and due to the finding that there is a statistical correlation between tumour response and resection rates across clinical studies [4]. Multimodality treatment combinations of systemic agents with liver surgery have been proffered as a means of improving tumour response rates and so improving the proportion of long term survivors of patients with metastatic colorectal cancer.

There has been much optimism that newly developed, expensive, biologically targeted agents may improve survival in this patient group, when combined with systemic chemotherapy, but complete radiological responses or cures remain exceedingly rare. Preliminary data from Phase III trials of chemotherapy and biologically targeted agents have not consistently shown statistically significant increases in response rates,nor the frequency of down-staging to resectability, over chemotherapy alone. There are two limitations of delivering neoadjuvant chemotherapy prior to liver metastectomy. Firstly, the development of pathological liver steatosis and fibrosis, which can occur with oxaliplatin-based chemotherapy or with irinotecan-based chemotherapy, and worsens with cumulative dosing. Secondly, the risk of disease relapse in the liver post-surgery, usually not at the resection site. Of those patients with liver-dominant or liver-only metastases, where the hepatic disease is unlikely to become resectable even with neoadjuvant systemic therapy, there is still a robust rationale for maximising local control of the liver disease, since up to $90 \%$ of patients with metastatic colorectal cancer ultimately die of liver failure [5]. Multiple loco-regional strategies are therefore under investigation to improve the outcome for patients with unresectable colorectal liver metastases, including radio-frequency ablation, hepatic arterial chemotherapy, cryotherapy and radio-embolization (RE), also known as selective internal radiotherapy (SIRT).

\section{Radio-embolization therapy}

RE with Ytrium-90 microspheres is a technique that has been developed to target multiple sites of disease within the liver as a form of brachytherapy. In contrast to surgical resection and radiofrequency ablation, its use is not limited by the number or site of liver metastases. TheraSpheres $^{\circledR}$ (MDS Nordion Inc., Kanata, Ontario, Canada) are glass microspheres and SIR-Spheres ${ }^{\circledR}$ (Sirtex Medical Ltd, Sydney, Australia) are resin microspheres, both of which contain the pure betaemitter, Ytrium-90, and have a mean diameter of 20-35 micrometers.

*Corresponding author: Dr. Ricky A. Sharma, Gray Institute, Department of Oncology, Old Road Campus Research Building, University of Oxford, Oxford OX3 7DQ, UK, E-mail: ricky.sharma@rob.ox.ac.uk

Received April 26, 2011; Accepted May 26, 2011; Published June 15, 2011

Citation: Hill EJ, Sharma RA (2011) Multi-modality Therapy of Hepatic Metastases from Colorectal Carcinoma: Optimal Combination of Systemic Chemotherapy with Radio-embolization. J Nucl Med Radiat Ther 2:108. doi:10.4172/21559619.1000108

Copyright: (c) 2011 Hill EJ, et al. This is an open-access article distributed unde the terms of the Creative Commons Attribution License, which permits unrestricted use, distribution, and reproduction in any medium, provided the original author and source are credited. 
SIR-spheres were first approved for use in Australia (1998) for the treatment of inoperable liver tumours and subsequently in the USA (2002) for the treatment of unresectable primary or metastatic liver tumours when combined with hepatic arterial fluoxuridine, and in the European Union (2002) for the treatment of primary and metastatic liver cancer. ${ }^{90} \mathrm{Y}$ glass microspheres are approved in Europe, India and Canada for the treatment of hepatic neoplasia. In the USA, ${ }^{90} \mathrm{Y}$ glass microspheres are approved for unresectable hepatocellular carcinoma.

The technique of RE involves an out-patient procedure in which a trans-femoral catheterisation is performed and, in the case of resin microspheres, approximately 40 to 80 million microspheres are injected into the arterial supply of the liver under fluoroscopic guidance. This treatment preferentially delivers a high dose of radiation to the liver tumour, whilst sparing much of the normal liver, by virtue of the fact that the tumour derives the majority of its blood supply from the hepatic artery and this characteristic ensures the injected microspheres lodge in the tumour microvasculature [6], whereas the normal liver predominantly receives its blood supply from the portal venous system. Many theoretical and clinical aspects of the utility of SIR-spheres for the treatment of metastatic colorectal cancer have been published, including reviews of the clinical response rate to treatment in the firstline, second-line and salvage settings [7-11].

Scientific rationale for combining RE with systemic chemotherapy currently used to treat metastatic colorectal cancer

It could be argued that some of the greatest breakthroughs in the treatment of solid malignancies have been made by administering chemotherapy and radiotherapy concurrently in the first-line setting, thus sparing patients from mutilating surgery [12-14]. There are a number of mechanisms by which drugs interact with radiation that can be exploited for therapeutic benefit [15]. The administration of potent systemic chemotherapy against metastatic colorectal cancer alongside radiation therapy targeted to disease in the liver exploits the mechanism of spatial co-operation, first described by Steel in 1979 [16]. Systemic chemotherapy targets macroscopic and microscopic disease outside the irradiated tissue whilst radiation therapy targets both macroscopic and microscopic disease in the liver, in the case of Ytrium 90 spheres, delivering a very high dose or radiation preferentially to the tumour whilst sparing normal liver tissue. As RE and chemotherapy have mostly non-overlapping toxicities, the combination of the two therapies theoretically should result in therapeutic gain, improving overall disease control and time to disease progression, whilst minimising unacceptable toxicity. Whereas the principle of spatial cooperation can potentially operate in any line of therapy, the highest response rates to chemotherapy and radiation in metastatic colorectal cancer are generally observed in the first line setting, with diminishing levels of response thereafter. Therefore, one might expect to see the best outcomes to be achieved from combining Ytrium 90 with systemic chemotherapy in the first line therapy of metastatic colorectal cancer.

Another mechanism by which chemotherapy drugs interact with radiation is by intrinsic radiosensitisation of tumour cells. At the cellular level, this means that the combined effect of administering chemotherapy and radiation together is greater than would be anticipated by merely adding together the independent anti-tumour effects expected from chemotherapy alone and radiation alone. In the case of metastatic colorectal cancer, all of the chemotherapy agents commonly used in the treatment of this disease have been demonstrated to be intrinsic radiosensitisers [17]. 5-Fluorouracil, which is the mainstay of colorectal cancer therapy, makes tumour cells more sensitive to the effects of radiation by inhibiting DNA repair of radiation-induced DNA damage, by producing double-stranded DNA breaks and killing cells in the S phase of the cell cycle, which is a relatively radioresistant phase of the cell cycle when DNA synthesis occurs $[18,19]$. Irinotecan is a chemotherapy drug that is approved for use in first line or subsequent therapy of metastatic colorectal cancer, either in combination with 5-fluorouracil or as a single agent. It is also a radiosensitiser in colon cancer cell lines [20]. It is a pro-drug for SN-38, which acts as an inhibitor of Topo-Isomerase 1, an enzyme involved in the production of single stranded DNA breaks during DNA replication. One mechanism by which irinotecan is thought to act as a radiosensitiser is the interaction between the DNA cleavable complex with replication machinery during DNA synthesis and by inhibition of sub-lethal radiation damage [20,21]. Oxaliplatin is a diaminocyclohexane compound which is a potent radiosensitiser in cells grown in vitro [22]. It has also been evaluated in combination with 5 -FU and radiation in vitro, where experiments revealed synergism in comparison to either radiation or the drugs alone [23]. The majority of the DNA damage caused by oxaliplatin is intrastrand DNA cross-links and monofunctional adducts, which it is believed the cell can repair relatively easily compared to interstrand DNA cross-links, which only represent a small proportion of the DNA lesions induced by oxaliplatin, but require repair of both DNA strands. It is thought that repair of the latter lesion, the interstrand crosslink, may overwhelm the cell's capacity to repair DNA damage in the context of irradiation, causing cells to arrest and potentially resulting in cell death. Additionally, the more common bulky DNA adducts induced by oxaliplatin also distort the DNA duplex, potentially blocking replication and transcription. Not surprisingly, oxaliplatin causes cell cycle arrest, which tends to be in the G2/M phase of cell cycle, which makes cell more radiosensitive. In summary, to capitalise on the potential enhancement of tumour response to Ytrium 90 radiation therapy by the mechanism of radiosensitisation it seems logical to co-administer radiosensitising chemotherapy, regardless of whether the multi-modality treatment is being used in first line therapy or a subsequent line of therapy.

\section{Optimal combinations of systemic chemotherapy with RE in metastatic colorectal cancer: First-line therapy}

A number of published studies of Ytrium-90 RE for metastatic colorectal cancer can be used to guide physicians in how to combine this treatment modality with systemic chemotherapy.

In 2001, Gray et al. [9] published a randomised controlled trial comparing hepatic arterial chemotherapy(HAC) with FUDR plus RE versus HAC with FUDR alone, in74 patients with nonresectable colorectal liver metastases, 63 of whom had not received prior chemotherapy for metastatic disease. All patients were treated with Fluoxuridine at $0.3 \mathrm{mg} / \mathrm{kg}$ body weight continuously for 12 days in 4-weekly cycles, for 18 cycles in total except in the case of discontinuation due to disease progression, unacceptable toxicity or patient choice. Patients receiving RE underwent a single treatment usually 4 weeks after insertion of the hepatic artery catheter. Analysis of the data collected from all 74 patients was reported and indicated a statistically significant improvement in radiological response rate from the addition of RE to HAC ( $17.6 \%$ vs $44 \% \mathrm{p}=0.01)$ and improvement in time to disease progression in the liver ( 9.7 months vs 15.9 months, $\mathrm{p}=0.01)$. The trial was not powered to detect a statistically significant difference in survival between the two groups, but a trend was observed towards improved survival in the group receiving RE and HAC, with improved survival in those living more than 15 months. There was no 
statistically significant difference in grade 3 and 4 toxicities between the two groups, nor impairment of quality of life in the group treated with RE. This trial identified the failure of disease control outside the liver as a significant problem, underlining the importance of systemic chemotherapy in this patient group. On separate analysis of data from the 63 patients who received treatment first-line, for the purpose of a Cochrane systematic review [24], the differences between the two groups were less pronounced, with response rates of $14 \%$ vs $37 \%$ (p0.051 ) in the HAC alone and HAC + RE groups respectively. There was no statistically significant improvement in progression free survival, nor overall survival observed as a result of the addition of RE to HAC in this patient group. It should be noted that 41 of the 63 patients had extra-hepatic disease, which may account for the failure of the study to demonstrate a survival benefit, but even on analysis of those 22 patients with hepatic-only disease, there was no statistically significant difference in progression-free survival between the two groups.

The second randomised trial comparing chemotherapy and RE against chemotherapy alone for patients with colorectal metastases in the first-line setting was published by Van Hazel et al. [10] in 2004. A Phase II randomised trial of systemic 5FU and Leucovorin plus SIRT vs 5FU/Leucovorin alone was conducted in 21 patients, five of whom had extra-hepatic metastases ( 2 in the combination group and 3 in the chemotherapy only group). Consistent with the Mayo regime that was used widely at that time, all patients were allocated to receive 5 -FU $425 \mathrm{mg} / \mathrm{m}^{2} /$ day plus Leucovorin $20 \mathrm{mg} / \mathrm{m}^{2}$ daily for 5 days, in 4 weekly cycles until the development of toxicity requiring cessation or disease progression; eleven of the patients were randomised to receive $\mathrm{RE}$ on the third or fourth day of the second cycle of chemotherapy. The study found that the RECIST response rate was better in the combination treatment group than the chemotherapy alone group, ten out of eleven patients demonstrating a partial response in the former group compared to none of the ten receiving chemotherapy alone. The median time to progression was significantly different between the two groups, 18.6 months in the combination group compared to 3.6 months in the chemotherapy group $(\mathrm{p}<0.0005)$ and median survival was 29.4 months vs 12.8 months $(\mathrm{p}=0.021)$ respectively. Grade 3 or 4 toxicity was also greater in the combination group but no statistically significant difference in quality of life was detected. One criticism of this study is that the sample size was small, with 2 patients in the chemotherapy-only arm not receiving any chemotherapy due to deterioration and patients in the combination group receiving more cycles of chemotherapy than those in the chemotherapy group [24]. Analysis of the data from those fifteen patients with no extra-hepatic disease in this trial by Townsend et al. [24] revealed a radiological response rate of $78 \%$ in the combination group and $0 \%$ in the chemotherapy group, with a median PFS of 19.1 months in the former group and 4.9 months in the latter group $(\mathrm{CI}$ : 0.06-0.91). The median survival of those with no extra-hepatic disease was 31.9 months vs 13.8 months (CI: 0.06-0.99). In summary, whilst the results of this trial are favourable towards the addition of RE to systemic chemotherapy, the small number of patients makes it difficult to draw definitive conclusions. Additionally, the systemic chemotherapy, used as first line therapy in this trial is no longer in widespread use. Overall, this study was a key step forward to show safety and proof-of-principle for combining RE with radiosensitising chemotherapy, and paved the way for subsequent first-line studies using oxaliplatin or Irinotecan in combination with $5 \mathrm{FU}$.

Oxaliplatin in combination with 5FU/LV was adopted in the late 1990 s as a significant new combination chemotherapy treatment for advanced or metastatic colorectal cancer. The FOLFOX4 regimen consists of bimonthly administration of Oxaliplatin $85 \mathrm{mg} / \mathrm{m}^{2}$ with standard LV/5FU, but a slightly different drug sequence (e.g. OxMdG) is often given in certain counties such as the UK. A number of large scale studies using FOLFOX or its variants have consistently yielded RECIST response rates of 50-60\%, progression-free survival of 8-9 months and median survival of 16-18 months [25]. Sharma et al. [26] reported promising results from a Phase I-II trial of FOLFOX chemotherapy in combination with RE for patients with unresectable colorectal liver metastases in the first line setting. Twenty patients were treated with oxaliplatin at escalating doses of $30-85 \mathrm{mg} / \mathrm{m}^{2}$ and full dose $5 \mathrm{LV} / 5 \mathrm{FU}$ for Cycle 1-3 and full dose FOLFOX 4 for cycles 4-12. The primary endpoint of the study was toxicity and the dose limiting toxicity was demonstrated to be G3/4 neutropenia(12 patients) with a maximum tolerated dose of oxalipltin $60 \mathrm{mg} / \mathrm{m}^{2}$ for cycles $1-3$. The combination treatment was generally well tolerated and 18 of the 20 patients $(90 \%)$ demonstrated a partial RECIST response to treatment; two (10\%) had stable disease. Two (10\%) of the patients responded to therapy sufficiently that they were able to undergo a partial hepatic resection. The median progression-free survival was 9.3 months overall, and 14.2 months in the seven patients with liver-only metastases, suggesting that the addition of RE might be most beneficial in this patient group. The overall median time to progression in the liver was 12.3 months.

This phase I-II study has paved the way for larger scale randomised phase III trials of oxaliplatin and 5FU with or without RE in the first line therapy of patients with liver-dominant metastatic colorectal cancer. Two studies are currently open to recruitment: the international SIRFLOX study, which is a randomised comparison study of FOLFOX6 plus SIR-spheres versus FOLFOX6 alone as first line treatment in patients with non-resectable liver metastases from primary colorectal cancer; and the UK National Cancer Research Network FOXFIRE trial, an open label randomised trial of 5-FU, Oxaliplatin and Folinic acid +/- Interventional radioembolisation as first line treatment for patients with unresectable liver only or liver-predominant metastatic colorectal cancer. In combination, these two trials aim to recruit 810 patients in total; data from the two trials will be pooled to analyse the primary endpoint of overall survival at 2-3 years of follow-up, as well as secondary endpoints including progression free-survival, response rate and quality of life. The FOXFIRE trial will also analyse health economics. It should be noted that these trials have attempted to define the concept of "liver-dominant" disease: The context of the FOXFIRE and SIRFLOX trials in treatment of mCRC is shown in Figure 1. Unless a significant amount of post-trial cross-over occurs in the subject group, it is hoped that the results of these large trials will definitively answer the question whether the addition of SIRT to first line chemotherapy provides survival benefit over giving chemotherapy alone.

\section{Clinical rationale for combining $\mathrm{RE}$ with chemotherapy for second and subsequent lines of therapy}

There is evidence to support the view that the combination of Ytrium $90 \mathrm{RE}$ with chemotherapy provides clinical benefit to patients with colorectal liver metastases in the second or subsequent lines of therapy.

In an important study of a radiosensitising drug other than oxaliplatin, Van Hazel et al. [27] perfomed a Phase I dose escalation study using single agent Irinotecan and RE in twenty-five patients with liver only or liver-dominant colorectal metastases who were refractory to 5-FU but had never received Irinotecan previously. Patients were treated with Irinotecan at escalating doses between 50 and $100 \mathrm{mg} /$ $\mathrm{m}^{2}$ Days 1 and 8 of a 21 day cycle for 2 cycles, with RE administered during cycle 1 and subsequently received full dose Irinotecan at 100 $\mathrm{mg} / \mathrm{m}^{2}$ Days 1 and 8 for cycles 3 to 9 . The trial demonstrated that the 


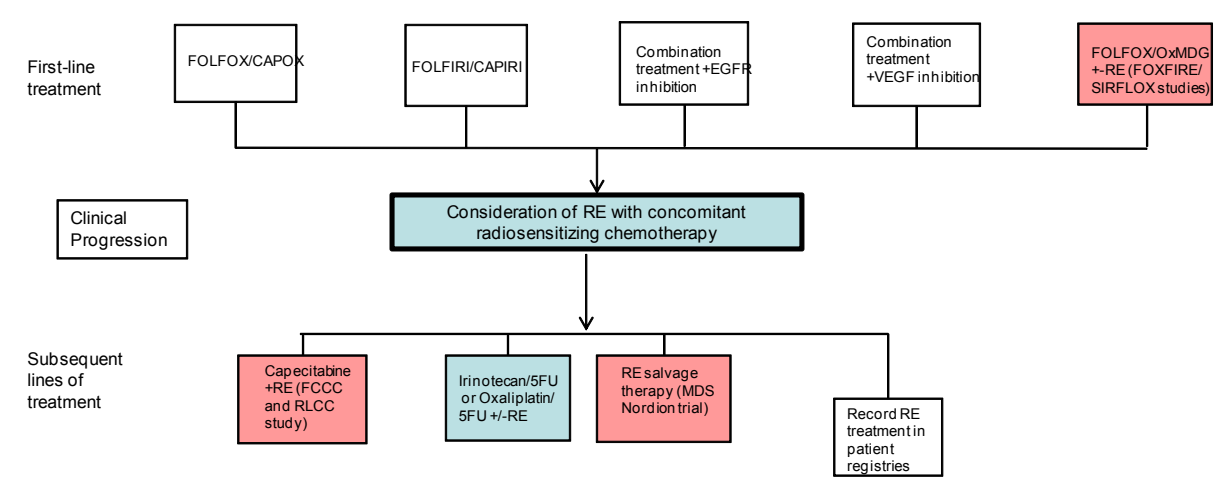

Figure 1: Potential points of integration of RE into the treatment pathway of patients with liver-only or liver-dominant unresectable colorectal liver metastases. Figure adapted from reference [17]. The shaded boxes show clinical trials testing RE with systemic therapy. Abbreviations used: CAPIRI, capecitabine and irinotecan; CAPOX, cetuximab, oxaliplatin and capecitabine; FCCC, Fox Chase Cancer Center; 5-FU, 5-fluorouracil; FOLFIRI, 5-fluorouracil, leucovorin and irinotecan; FOLFOX, 5-fluorouracil, leucovorin and oxaliplatin; RE, radioembolization; RLCC, Robert H. Lurie Comprehensive Cancer Center. An example of a patient registry in development is: http://www.sirtex.com/content.cfm?sec=usa\&MenulD=1120\&ID=F4CCC1C2

combination of Irinotecan as second line chemotherapy with RE was not only safe, but appeared to be efficacious. The maximum tolerated dose was not reached and therefore the recommended dose of Irinotecan for combination with RE was $100 \mathrm{mg} / \mathrm{m}^{2} \mathrm{D} 1$ and 8,3 weekly. In the study, $48 \%$ of patients were observed to have a partial radiological response to therapy with a median progression free survival of 6 months and median survival of 12.2 months. Although not a randomised comparison, these statistics certainly compare favourably with those reported for other irinotecan based regimes used in this clinical setting [28-31].

RE plus chemotherapy has also been demonstrated as a valuable treatment strategy for patients who have progressed on standard systemic anti-cancer chemotherapy. The most significant study in this setting is the recent report by Hendlisz et al. [32]. This was a Phase III study of 46 patients with chemorefractory liver-only colorectal metastases who were randomised to receive either infusional 5FU or infusional 5FU with the additional of RE. Patients in the control arm received 5FU $300 \mathrm{mg} / \mathrm{m}^{2} \mathrm{~d} 1-14$ every 3 weeks and patients in the RE arm received 5FU $225 \mathrm{mg} / \mathrm{m}^{2} \mathrm{D} 1-14$ for 1 cycle then $300 \mathrm{mg} / \mathrm{m}^{2}$ Day114 in subsequent cycles. The primary endpoint of the trial was Time to Progression within the Liver (TTLP) and crossover was permitted for patients developing progressive disease. The trial reached its primary endpoint by showing that RE significantly extended TTLP by 3.4 months (from 2.1 to 5.5 months; hazard ratio 0.38 , $95 \%$ confidence interval [CI 0.20 to $0.72 ; \mathrm{p}=0.003$ ). It also showed a statistically significant extension in Time to Progression (TTP) overall, from 2.1 to 4.6 months (hazard ratio $0.51,95 \% \mathrm{CI} 0.28-0.94 ; \mathrm{p}=0.03$ ). The disease control rate (partial response and stable disease) was significantly better in the RE arm compared with the control arm at $86 \%$ vs $35 \%$ $(\mathrm{p}=0.001)$ respectively. Grade 3 and 4 toxicity rates were higher in the control arm, but this difference was not statistically significant. The median overall survival of the groups combined was 8.7 months, with no statistically significant difference between the two groups. This may partly be explained by the fact that twenty five out of forty four patients whose data was analysed received further treatment for their cancer on progression of their disease, including 10 out of 25 patients in the control arm who subsequently received RE.

Although it may be optimal to combine RE with radiosensitising systemic therapy whenver possible and safe, there are also studies which suggest that Ytrium 90 RE without concurrent chemotherapy treatment is a beneficial treatment in the salvage setting. Ricke et al. [33] perfomed a Phase II trial of RE alone as salvage therapy in 29 patients with extensive colorectal liver metastases, with a matchedpair analysis in comparison against a further 29 control patients who received best supportive care. All of the patients had been heavily pre-treated with chemotherapy (median of 4 lines of chemo in the RE group and 3 lines in the control group). Median PFS was significantly prolonged in patients receiving SIR-Spheres microspheres compared with those in the BSC cohort (5.5 vs. 2.1 months; $P<0.001)$. The median overall survival was significantly longer for the patients receiving $\mathrm{RE}$ compared with controls ( $8.3 v s .3 .5$ months; $P<0.001)$. This benefit was clearly evident at 3 months ( $97 \%$ vs. 59\% survival) and was sustained through 12 -months follow up ( $24 \%$ vs. $0 \%$ survival). A multivariate Cox proportional hazard model analysis was used to correlate a range of variables with outcome, but the only prognostic variable was the lack of RE treatment $(p<0.01)$. Similarly, Cosimelli et al. [34] conducted a Phase II prospective trial of Ytrium RE in 50 patients who had chemorefractory colorectal liver metastases, all having received more than 3 lines of chemotherapy, including one oxaliplatin-containing regime and one irinotecan-containing regime. The primary endpoint of this trial was objective response rate, determined to be $24 \%$ ( $2 \%$ complete responses and $22 \%$ partial responses), with a further $24 \%$ of patients demonstrating stable disease after therapy. Two of the responding patients were able to undergo a potentially curative liver resection. The median overall survival was 12.6 months, with a statistically significant difference between the survival of responders and non-responders ( 16 months vs. 8 months; $\mathrm{p}=0.0006$ ). However, the median Time to progression was only 3.7 months, much shorter than that observed in the first-line setting when combined with chemotherapy(18.6 months [10]).Collectively, these trials suggest that $\mathrm{RE}$ without chemotherapy is an appropriate treatment in the salvage setting in patients with liver dominant colorectal metastases who have received multiple lines of chemotherapy previously.

Although RE can be used alone or in combination with systemic chemotherapy in the salvage setting, the view that Ytrium $90 \mathrm{RE}$ combined with radiosensitising chemotherapy at an earlier stage in a patient's treatment may be preferable is reinforced by a meta-analysis of 18 trials involving Ytrium $90 \mathrm{RE}$ [35]. This determined that the response rate to RE and chemotherapy in the salvage setting was $79 \%$, but the researchers found it to be over $90 \%$ in the first line setting, irrespective of whether 5-FU was used alone or in combination with oxaliplatin or irinotecan. 

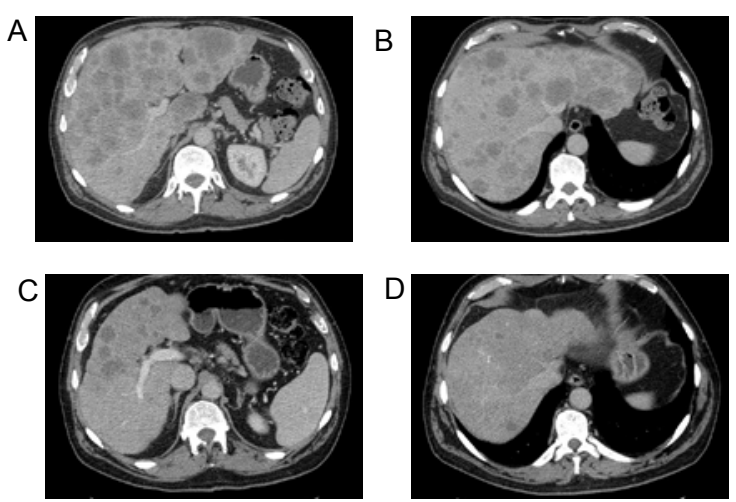

Figure 2: Axial CT scan of a patient with metastatic colorectal cancer treated with systemic FOLFOX chemotherapy with RE administered via right and left hepatic arteries on day 3 of cycle 2 . Panels A and B show pre-chemotherapy extent of disease, quantified at $65 \%$ replacement of liver tissue by malignant disease. Panels $C$ and $D$ show the extent of tumour regression 10 weeks after the RE procedure.

\section{Guidelines for combining RE with systemic chemotherapy}

Based on the current evidence base, rigid recommendations cannot be advocated regarding criteria to combine RE with systemic chemotherapy in metastatic colorectal cancer in different lines of treatment of metastatic disease. The following are important points to consider in guiding physicians to combine these treatments optimally.

An important consideration is that patient selection for RE should be made only following thorough radiological assessment regarding the extent of metastatic disease within and outside the liver. As analysis of the randomised trials published by Gray [9] and Van Hazel [10] suggested, the presence of a significant burden of extra-hepatic metastases may limit the potential benefit obtained from RE in some patients. Despite this, the trials described above demonstrate that RE may be very appropriate therapy in patients with unresectable liver metastases and limited extra-hepatic metastases, as the hepatic disease is highly likely to be life-limiting for most patients. Indeed, the case for $\mathrm{RE}$ is strongest for patients with liver-only disease or liver-dominant disase, where the combination of chemotherapy and radiotherapy has been shown to result in long-term disease control or down-sizing to surgery or ablative therapy in some patients. This case is exemplified by a series of 46 patients with liver-only metastases, derived from mostly colorectal and breast primary tumours, who received a single treatment with RE [36]. In five patients, the liver disease was sufficiently downsized for subsequent Radiofrequency Ablation (RFA) of the residual lesions to be performed. It is notable that all 5 of these patients had a complete radiological response to RFA. Since it is not currently clear which patients benefit most from RE therapy, it is desirable to deliver RE and chemotherapy in the context of a clinical trial, when available. Figures 1 and 2 show how RE and chemotherapy are being integrated in different lines of therapy, based on the contemporary design of clinical trials.

Which drug or combination of drugs should be given with yttrium -90 for optimal radiosensitisation? It should be noted that, since the biological mechanism of radiosensitisation (as discussed above) may be independent of the mechanism of anti-cancer efficacy of the same drugs used without concomitant radiotherapy, there is a scientific rationale for using a radiosensitising chemotherapy drug that the patient has previously received and may even have previously shown "tumor resistance" to. Oxaliplatin, 5-FU and irinotecan are all radiosensitisers, and the selection of radiosensitising chemotherapy in clinical practice should be guided by the dosing regimes shown to be safe with RE $[26,27,32]$ rather than by the need to control microscopic or macroscopic disease outside the liver. Following the administration of radiosensitising chemotherapy with RE, the patient can subsequently receive a systemic regime which may offer further survival benefit.

Currently, insufficient evidence exists to routinely recommend the addition of Ytrium-90 RE to systemic chemotherapy in the first line setting. Patients who wish to receive RE as first-line therapy for their metastatic colorectal cancer, and who fulfil the inclusion criteria for the clinical trials, should participate in the SIRFLOX and FOXFIRE studies. These clinical trials are currently recruiting patients in the UK, Europe, Australia, New Zealand and the USA. Since over 250 patients have already been recruited to these studies, it is anticipated that recruitment to both studies will be completed by 2014 and survival data should be available in 2017 .

The practice at our institution is to offer oxaliplatin and 5-FU chemotherapy (in the dosing regime shown to be safe in reference [26]) with RE as second line or subsequent line therapy for patients with liver-dominant or liver-only metastases from colorectal cancer, regardless of their previous chemotherapy history. In patients who have experienced unacceptable toxicity with oxaliplatin (e.g. persistent peripheral neuropathy), RE can be combined with infusional 5FU [32] or with irinotecan [27], using the dosing regimes of chemotherapy shown to be safe. Our current practice is to administer RE on day 2, 3 or 4 of cycle 1 and to administer 6 weeks of chemotherapy in total for radiosensitisation. Following 6 weeks of therapy from the date of $\mathrm{RE}$, chemotherapy can be switched to an alternative systemic regime to optimally manage extra-hepatic disease.

\section{Conclusions}

The combination of RE and systemic chemotherapy can downstage a significant proportion of patients with unresectable liver metastases to render them amenable to potentially curative surgery or ablation. Furthermore, since liver disease progression is the principal cause of death in patients with metastatic colorectal cancer, there is a potential survival benefit to be gained from local control of liver metastases in the context of limited extrahepatic disease. The evidence base for combining RE and chemotherapy in the first-line treatment of metastatic colorectal cancer is robust enough for 2 large-scale phase III trials to have been initiated to test the hypothesis that the greatest clinical benefit from RE may be achieved from this combination therapy at an early timepoint in a patient's disease course. These randomised trials should provide high quality evidence regarding whether this strategy improves the overall survival of patients with liver-dominant or liver-only unresectable liver metastases, and which subgroups of patients benefit most. In second-line and subsequent lines of therapy for metastatic colorectal cancer, we recommend that the combination of RE and systemic chemotherapy should be performed based on the principles of optimal intrinsic radiosensitisation of the liver metastases rather than by the concept of spatial cooperation, i.e. the need to control microscopic or macroscopic disease outside the liver. Following the administration of 6 weeks of radiosensitising chemotherapy with RE, patients can subsequently receive any systemic regime for further survival benefit.

\section{Acknowledgements}

The authors are funded by Oxfordshire Health Services Research Committee, the Higher Education Funding Council for England and the NIHR Biomedica Research Centre Oxford. 
Citation: Hill EJ, Sharma RA (2011) Multi-modality Therapy of Hepatic Metastases from Colorectal Carcinoma: Optimal Combination of Systemic Chemotherapy with Radio-embolization. J Nucl Med Radiat Ther 2:108. doi:10.4172/2155-9619.1000108

\section{References}

1. US SEER Data.www.seer.cancer.gov, 2005

2. Delaunoit T, Alberts SR, Sargent DJ, Green E, Goldberg RM, et al. (2005) Chemotherapy permits resection of metastatic colorectal cancer: experience from Intergroup N9741. Ann Oncol 16: p. 425-429.

3. Nordlinger B, Van Cutsem E, Rougier P, Köhne CH, Ychou M, et al. (2007) Does chemotherapy prior to liver resection increase the potential for cure in patients with metastatic colorectal cancer? A report from the European Colorectal Metastases Treatment Group. Eur J Cancer 43: 2037-2045.

4. Folprecht G, Grothey A, Alberts S, Raab HR, Köhne CH (2005) Neoadjuvan treatment of unresectable colorectal liver metastases: correlation between tumour response and resection rates. Ann Oncol 16: 1311-1319.

5. Nagorney DM, Gigot JF (1996) Primary epithelial hepatic malignancies: etiology, epidemiology, and outcome after subtotal and total hepatic resection. Surg Oncol Clin N Am 5: 283-300.

6. Ho S, Lau WY, Leung TW, Chan M, Chan KW, et al. (1997) Tumour-to-normal uptake ratio of $90 \mathrm{Y}$ microspheres in hepatic cancer assessed with $99 \mathrm{Tcm}$ macroaggregated albumin. Br J Radiol 70: 823-828.

7. Kennedy AS, Coldwell D, Nutting C, Murthy R, Wertman DE Jr, et al. (2006) Resin 90Y-microsphere brachytherapy for unresectable colorectal live metastases: modern USA experience. Int J Radiat Oncol Biol Phys 65: 412425

8. Stubbs RSI, Brien O, Correia MM (2006) S Selective internal radiation therapy with $90 \mathrm{Y}$ microspheres for colorectal liver metastases: single-centre experience with 100 patients. ANZ J Surgery 76: 696-703.

9. Gray B, Van Hazel G, Hope M, Burton M, Moroz P, et al. (2001) Randomised trial of SIR-Spheres plus chemotherapy vs. chemotherapy alone for treating patients with liver metastases from primary large bowel cancer. Ann Oncol 12: $1711-1720$.

10. Van Hazel G, Blackwell A, Anderson J, Price D, Moroz P, et al. (2004) Randomised phase 2 trial of SIR-Spheres plus fluorouracil/leucovorin chemotherapy versus fluorouracil/leucovorin chemotherapy alone in advanced colorectal cancer. J Surg Oncol 88: 78-85.

11. Gray BN, Anderson JE, Burton MA, van Hazel G, Codde J, et al. (1992) Regression of liver metastases following treatment with yttrium-90 microspheres. Aust N Z J Surg 62: 105-10.

12. Rose PG (2002) Chemoradiotherapy for cervical cancer. Eur J Cancer 38: 270278.

13. Pignon JP, Bourhis J, Domenge C, Designé L (2000) Chemotherapy added to locoregional treatment for head and neck squamous-cell carcinoma: three meta-analyses of updated individual data. $\mathrm{MACH}-\mathrm{NC}$ Collaborative Group. Meta-Analysis of Chemotherapy on Head and Neck Cancer. Lancet 355 : 949955.

14. Sebag-Montefiore D (2006) Developments in the use of chemoradiotherapy in rectal cancer. Colorectal Dis 8: 14-17.

15. Bentzen SM, Harari PM, Bernier J (2007) Exploitable mechanisms for combining drugs with radiation: concepts, achievements and future directions. Nat Clin Pract Oncol 4: 172-180.

16. Steel GG, Peckham MJ (1979) Exploitable mechanisms in combined radiotherapy-chemotherapy: the concept of additivity. Int J Radiat Oncol Bio Phys 5: 85-91.

17. Nicolay NH, Berry DP, Sharma RA (2009) Liver metastases from colorecta cancer: radioembolization with systemic therapy. Nat Rev Clin Oncol 6: 687697.

18. Lawrence TS, Davis MA, Maybaum J (1994) Dependence of 5-fluorouracilmediated radiosensitization on DNA-directed effects. Int J Radiat Oncol Biol Phys 29: 519-523.

19. Yoshioka A, Tanaka S, Hiraoka O, Koyama Y, Hirota Y, et al. (1987) Deoxyribonucleoside triphosphate imbalance. 5-Fluorodeoxyuridine-induced DNA double strand breaks in mouse FM3A cells and the mechanism of cell death. J Biol Chem 262: 8235-8241.

20. Chen AY, Okunieff P, Pommier Y, Mitchell JB (1997) Mammalian DNA topoisomerase I mediates the enhancement of radiation cytotoxicity by camptothecin derivatives. Cancer Res 57: 1529-1536.

21. Illum $H$ (2011) Irinotecan and radiosensitization in rectal cancer. Anticancer Drugs 22: 324-329.

22. Blackstock AW (1999) 202 Oxaliplatin: In vitro evidence of its radiation sensitizing activity -- preclinical observations relevant to clinical trials. Intern $J$ Rad Oncol 45: 253-254.
23. Kjellström J, Kjellén E, Johnsson A (2005) In vitro radiosensitization by oxaliplatin and 5-fluorouracil in a human colon cancer cell line. Acta Oncol 44 $687-693$.

24. Townsend A, Price T, Karapetis C (2009) Selective internal radiotherapy fo liver metastases from colorectal cancer. Cochrane Database Syst Rev 7 : CD007045.

25. Louvet C, de Gramont A (2003) Colorectal cancer: integrating oxaliplatin. Curr Treat Options Oncol 4: 405-411.

26. Sharma RA, Van Hazel GA, Morgan B, Berry DP, Blanshard K, et al. (2007) Radioembolization of Liver Metastases From Colorectal Cancer Using Yttrium-90 Microspheres With Concomitant Systemic Oxaliplatin, Fluorouracil, and Leucovorin Chemotherapy. J ClinOncol 25: 1099-1106.

27. van Hazel GA, Pavlakis N, Goldstein D, Olver IN, Tapner MJ, et al. (2009) Treatment of Fluorouracil-Refractory Patients With Liver Metastases From Colorectal Cancer by Using Yttrium-90 Resin Microspheres Plus Concomitant Systemic Irinotecan Chemotherapy. J ClinOncol 27: 4089-4095.

28. Cunningham D, Pyrhönen S, James RD, Punt CJ, Hickish TF, et al. (1998) Randomised trial of irinotecan plus supportive care versus supportive care alone after fluorouracil failure for patients with metastatic colorectal cancer. Lancet 352: 1413-1418.

29. Seymour MT, Maughan TS, Ledermann JA, Topham C, James R, et al. (2007) Different strategies of sequential and combination chemotherapy for patients with poor prognosis advanced colorectal cancer (MRC FOCUS): a randomised controlled trial. Lancet 370: 143-152.

30. Schoemaker NE, Kuppens IE, Moiseyenko V, Glimelius B, Kjaer M, et al. (2004) A randomised phase II multicentre trial of irinotecan (CPT-11) using fou different schedules in patients with metastatic colorectal cancer. $\mathrm{Br} \mathrm{J}$ Cancer 91: 1434-1441.

31. Mabro M, Artru P, André T, Flesch M, Maindrault-Goebel F, et al. (2006) A phase II study of FOLFIRI-3 (double infusion of irinotecan combined with LV5FU) afte FOLFOX in advanced colorectal cancer patients. Br J Cancer 94: 1287-1292.

32. Hendlisz A, Van den Eynde M, Peeters M, Maleux G, Lambert B, et al. (2010) Phase III Trial Comparing Protracted Intravenous Fluorouracil Infusion Alone or With Yttrium-90 Resin Microspheres Radioembolization for Liver-Limited Metastatic Colorectal Cancer Refractory to Standard Chemotherapy. J Clin Oncol 28: 3687-3694.

33. Ricke J (2009) Extensive Liver-Dominant Colorectal (Crc) Metastases Failing Multiple Lines of Systemic Chemotherapy Treated by 90y Radioembolisation: A Matched-Pair Analysis. Annal Oncol 20: 17-17.

34. Cosimelli M, Golfieri R, Cagol PP, Carpanese L, Sciuto R, et al. (2010) Multi-centre phase II clinical trial of yttrium-90 resin microspheres alone in unresectable, chemotherapy refractory colorectal liver metastases. $\mathrm{Br} \mathrm{J}$ Cancer 103: 324-331.

35. Vente MA, Wondergem M, van der Tweel I, van den Bosch MA, Zonnenberg BA, et al. (2009) Yttrium-90 microsphere radioembolization for the treatment of liver malignancies: a structured meta-analysis. Eur Radiol 19: 951-959.

36. Hoffmann RT, Jakobs TF, Kubisch CH, Stemmler HJ, Trumm C, et al. (2010) Radiofrequency ablation after selective internal radiation therapy with Yttrium90 microspheres in metastatic liver disease-Is it feasible? Eur J Radiol 74: 199 205 